\section{Exit exceptionalism: mental disease is like any other medical disease}

In their well-written editorial, ${ }^{1}$ Malla, Joober and Garcia argue that mental disease is different from other medical disease. Although interesting, their arguments hardly undermine the claim that mental disease is like any other medical disease. Moreover, in their eagerness to demarcate psychiatry from somatic medicine they miss a crucial opportunity for psychiatry.

Their main arguments are as follows: mental disease involves the self, it cannot be explained in neurobiological terms, it is not situated in an organ, and biological explanations are not helpful (e.g., for reducing stigma).

Here is where their arguments fail. Mental disease involves the self. The authors argue that the hallmark defining features of mental disorders are "the changes in how the patients feel, think and act and how these changes affect their relation to themselves and to others." Although perfectly right, these characteristics are not unique to mental diseases. A person who is in pain and distress due to cancer can have a significantly altered way of thinking, feeling and relationship to others and him/herself. The point is that all diseases involve the self. Disease is a basic attack on the human being, not only on our mental selves. Disease alters who we are and who we can be - physically, morally and socially - not only mentally. A person with an incapacitating infection cannot pick up his children from school, be a devoted husband, a caring son or a dedicated employee.

Mental disease cannot be explained in neurobiological terms, particularly not "many of the behaviours and experiences that constitute the core presentations of mental disease." Although the authors may be perfectly right in this claim, it does not bolster the uniqueness of mental disorders. A wide range of other diseases cannot be explained in neurological (or other biological, physiological or bimolecular) terms either. Using causal explanations as a sine qua non for disease would eliminate a wide range of diseases.

Mental disease cannot be situated in an organ. Again, there are numerous diseases that cannot be situated in a specific organ. Neurofibromatosis is but 1 example. The point is that not all diseases are organ-specific, so this is hardly unique for mental disease. The same goes for the argument that biological explanations are not helpful. Malla, Joober and Amparo may very well be right that biological explanations may have negative consequences for (the self-conception of) some people with mental diseases. First, the opposite may also be the case (i.e., it may have good consequences). Second, this hardly buttresses the uniqueness of mental diseases. On the contrary, it points to deficiencies in "somatic medicine" not paying attention to self-inflicting consequences of any disease. Third, the history of psychiatry is full of misguided and unhelpful explanations.

It is important to note that I am not saying that there are no good arguments for the uniqueness of mental diseases. I have argued only that the arguments presented by opinion leaders in the field may not do the job. They do not demonstrate the uniqueness of mental diseases. The main flaw, as I see it, is not that they do not address core aspects of mental diseases. On the contrary, I do think that the self-affecting aspects the authors refer to are essential to mental diseases. However, the mistake is to make them exclusive to mental disease. All disease, mental or otherwise, appears to affect the human self.

The danger of exceptionalism: One reason to undermine unwarranted exceptionalism in psychiatry is the harm that it can do. Historically psychiatry has allowed too much, for example, resulting from slack demands for rigour and evidence. No doubt, selfaffecting aspects of disease are core to psychiatry, but they are not unique.
This is where psychiatry could make a significant contribution to medicine in general: teaching us to pay attention to the self-affecting aspects of disease. Hence, mental disease is like any other medical disease. All diseases are self-afflicting. This makes psychiatry crucial for understanding and handling all diseases. Don't miss this pivotal opportunity!

\section{Bjørn Hofmann}

The Centre of Medical Ethics at the University of Oslo, and Department for Health, Technology and Social Sciences at the University College of Gjøvik, Norway

DOI: $10.1503 / j p n .150262$

\section{Reference}

1. Malla A, Joober R, Garcia A. Mental illness is like any other medical illness: a critical examination of the statement and its impact on patient care and society. J Psychiatry Neurosci 2015;40:147-50.

\section{Author response}

We are pleased to see the critical commentary by Hofmann and we provide a brief response to this critique.

1 . The first concern seems to be related to our argument that mental illness involves the self in different ways than other medical disorders, such as diabetes. We have clearly argued that the difference is that mental illness affects the very core of self and that it is in essence a disorder of the self. Of course other medical disorders, including cancer - an example used by Hoffman - indeed affect the self, but they are not disorders of the self and do not affect the very core of what constitutes self.

2. The second argument is regarding the neurological basis of mental disorders. We have not claimed in our editorial that there is no neurobiological basis of mental illness. On the contrary, we have acknowledged the important contribution made by neurosciences in explaining 
many of the "uniquely human phenomena, such as memory, thinking," etc. First, we have emphasized 2 points in relation to neurobiology. One is that at this stage many of the experiences described by patients with mental disorders are not explained by neurobiology and that even if 1 day they were, we would still need to understand mental illness in psychological terms. ${ }^{1}$ The other issue is related to the extent to which predominantly neurobiological explanations of mental disorders have failed to have any positive impact on reducing stigma among the public, contrary to expectations.

3. In response to the argument about "the danger of exceptionalism," we had clearly indicated at the beginning of the article that it was not our intention to argue for or against the biological basis of mental illness, but rather "to examine the clinical and public utility of presenting a dominant neurobiological model of mental illness." We do not believe this, in any way, makes mental illness an exception, but rather offers an evidence-based approach to what the public needs to know about mental illness. This should be based on all the facts and not on a selective explanatory model.

We hope that these clarifications address some of the issues raised in the letter from Hofmann.

Ashok Malla, Ridha Joober, Amparo Garcia From the Department of Psychiatry, McGill University, Montreal, Que., Canada.

DOI: 10.1503/jpn.150319

\section{Reference}

1. Kendler KS. The structure of psychiatric science. Am J Psychiatry 2014;171:931-8. 